\title{
Screening in dry two-dimensional foams
}

\author{
S. J. Cox, ${ }^{\star a b}$ F. Graner ${ }^{b}$ and M. F. Vaz \\ Received 18th February 2008, Accepted 23rd May 2008 \\ First published as an Advance Article on the web 27th June 2008 \\ DOI: 10.1039/b802792g
}

We perform numerical simulations of point-like deformations in a dry two-dimensional foam using the Surface Evolver software. We study perturbations which are infinitesimal or finite, isotropic or anisotropic, and we either conserve or do not conserve the number of bubbles. We measure the displacement fields around the perturbation. Changes in pressure decrease exponentially with the distance to the perturbation, indicating a screening over a few bubble diameters.

\section{Introduction}

A dry foam is a discrete material, made of polyhedral gas bubbles separated by thin walls of a continuous liquid phase. Foams act as elastic solids for small deformations, but when large strains are applied they flow like plastic solids and, at higher shear rate, like viscous liquids, ${ }^{1-3}$ applied stresses are relaxed by discrete rearrangement events that occur in the foam. Other changes are due to ageing, where some bubbles gain gas at the expense of others. In most cases of foam evolution, continuous changes, such as infinitesimal changes of bubble shapes and sizes, alternate with discontinuous processes. ${ }^{4}$

For instance, the length of an edge might decrease (or, conversely, it might stretch). If it vanishes, a neighbour-swapping event occurs, and a new edge is created; this is the topological T1 process $^{5}$ (its inverse is also a T1). Alternatively, an edge breakage leads to bubble coalescence, also called fusion (its inverse is a division). All these perturbations are anisotropic. On the other hand, when a bubble's area decreases, it is an isotropic perturbation (its inverse is a bubble inflation). If it vanishes, a reduction in the number of bubbles occurs: this is reminiscent of biological cell apoptosis, or of a topological T2 process, ${ }^{5}$ preceded by a number $n-3$ of T1s, where $n$ is the number of sides of the disappearing bubble (its inverse is a nucleation).

Each perturbation affects the neighbouring bubbles, over a certain range. This range has been the subject of various studies, with diverse motivations, all in two dimensions. The perturbation induced by a T1 has been measured in experiments ${ }^{6}$ and in simulations. ${ }^{7}$ The effect of changing the volume of a single bubble has also been studied, both in experiments ${ }^{8,9}$ and in simulations. ${ }^{10,11}$ A laser has been used to break the wall between bubbles ${ }^{12}$ to study coalescence.

An infinitesimal change is expected to have an infinitesimal effect on neighbouring bubbles. Conversely, finite discontinuities can have a finite effect on other bubbles. If these events are scarce enough, and spatially screened by the foam disorder, ${ }^{13}$ their

\footnotetext{
Institute of Mathematics and Physics, Aberystwyth University, Ceredigion, SY23 3BZ,UK.E-mail: foams@aber.ac.uk

${ }^{b}$ Laboratoire de Spectrométrie Physique, UMR5588, CNRS-Université Grenoble I, B.P. 87, Martin d'Hères Cedex, F-38402, France

'Instituto de Ciência e Engenharia de Materiais e Superficies and Departamento de Engenharia de Materiais, IST, UTL, Avenida Rovisco Pais, 1049-001 Lisboa, Portugal
}

effects on the foam are independent, and the foam can be treated as a continuous material ${ }^{14}$ at a large scale. The foam properties of insulation against sound and explosion partly derive from the screening. A question under debate is to determine under which conditions these processes correlate, triggering for instance a cascade of T1s.,6,9,11 This can lead to large-scale fluctuations such as avalanches. ${ }^{15-18}$

Over the last few years, "soft materials" such as foams, granular packings, pastes and biological tissues have attracted the attention of many authors. In all these materials, it is important to predict how changes in the structure will affect their mechanical properties. In particular, the role of disorder on perturbation screening has been studied in various discrete systems such as rigid grains and particles. ${ }^{19-27}$ It is not a priori clear that the results should apply to the deformable objects (bubbles) considered here.

In addition, the laser ablation referred to above is used in biological tissues, ${ }^{28}$ and cell division and apoptosis are also currently being studied, ${ }^{29}$ motivated by a desire to understand the mechanics of biological tissue.

To study the screening in foams, we focus on a 2D simulated dry foam with an isolated perturbation. Physically, this is relevant for a system that is initially formed by well-separated defects, that may eventually interact. We define screening to be an exponential decrease in a measured quantity away from the perturbation; this can be quantified by a characteristic length. Such an exponential decrease implies that the signal is soon very low, and at some places can no longer be detected. In experiments, the detection threshold depends on the experiment's noise, and a variable part of the exponential can be measured. In simulations, we can expect the noise (and thus the detection threshold) to be intrinsic to the cluster's disorder, and thus interesting to measure, plot and study, but not as interesting as the characteristic length.

Here, we simulate small continuous geometrical changes, then finite discontinuous topological processes. We either conserve or change the number of bubbles. We try different foam disorders and boundary conditions. We perform isotropic (scalar) perturbations, and anisotropic (tensorial) ones. Two types of measurements are extracted from the simulations: the displacement of each bubble (a vector), and the change in pressure $^{30}$ in each bubble, with respect to the initial (unperturbed) foam. 


\section{Method}

We create a disordered foam structure using a Voronoi construction based upon a random Poisson process for generating the cell centres. ${ }^{31}$ The cell areas $A$ are chosen based upon a randomly generated Weibull distribution: ${ }^{32}$

$$
f(A ; \beta, \lambda)=\frac{\beta}{\lambda}\left(\frac{A}{\lambda}\right)^{\beta-1} e^{-(A / \lambda)^{\beta}},
$$

where the parameter $\beta>1$ determines the area dispersity and the parameter $\lambda$ is chosen as $\lambda=1.115\langle A\rangle$, so that the peak of the distribution is close to $A=\langle A\rangle$. For the monodisperse case, each cell is set to have the same target area, rather than taking the limit $\beta \rightarrow \infty$. We also constructed ordered monodisperse foams consisting of 8 and 20 concentric shells of hexagons. ${ }^{33}$ The disorder of each foam is defined by the (dimensionless) variance of the area distribution:

$$
\operatorname{var}(A)=\frac{\left\langle(A-\langle A\rangle)^{2}\right\rangle}{\langle A\rangle^{2}},
$$

where \langle\rangle denotes an average over the whole foam.

Each foam is first equilibrated using the Surface Evolver software, ${ }^{34}$ using a mode in which each film is represented exactly as a circular arc and a value of surface tension $\gamma$ equal to one. Bubble pressures are found by the minimisation algorithm, defined and measured as the Lagrange multiplier of the volume constraint. T1 topological changes are automatically triggered when films shrink to a very small length during the equilibration

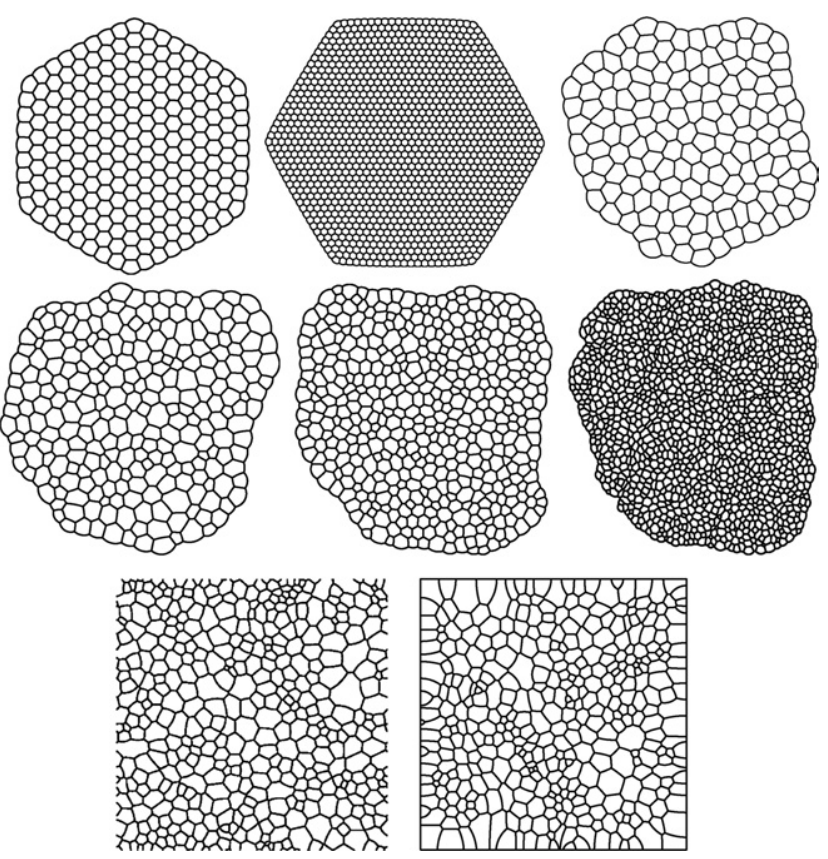

Fig. 1 Representative images of the foams used. Top row: monodisperse foams, both ordered (217 and 1261 bubbles) and disordered (150 bubbles). Middle row: polydisperse foams, in each case with almost the same value of polydispersity, $\operatorname{var}(A) \approx 0.12$, and 250,450 and 1400 bubbles, respectively. Bottom row: polydisperse foams without boundaries (periodic, 400 bubbles) and with boundaries ( 383 bubbles), respectively.
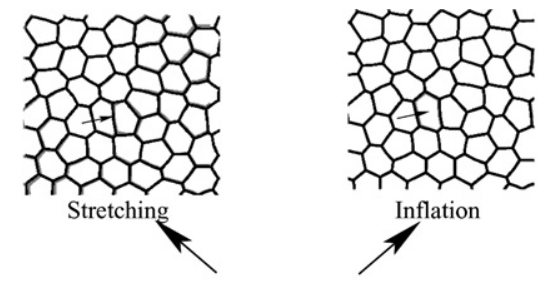

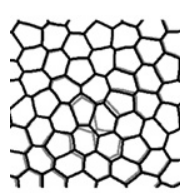

T1

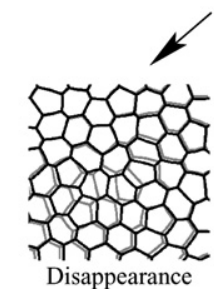

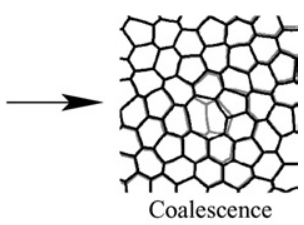

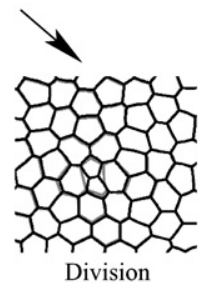

Fig. 2 The six types of distortion applied to the foams, illustrated here for the disordered monodisperse foam of 150 bubbles. Edge stretch (by $20 \%$ ), inflation (by $20 \%$ ), T1, coalescence, disappearance, division.

process. Some of the initial foam structures used are shown in Fig. 1 .

We then trigger changes to the structure as follows, illustrated in Fig. 2:

- An edge stretch is performed on the longest edge of the bubble nearest the centre of the cluster, by extending or reducing its length by a fraction $\delta L$ and fixing its endpoints.

- An inflation of the bubble nearest the centre of the cluster by a fraction $\delta A$.

- A $T 1$ is performed on the shortest edge of the bubble nearest the centre of the cluster.

- A disappearance event is triggered by removing the bubble nearest the centre of the cluster.

- A coalescence event is triggered by removing the longest edge of the bubble nearest the centre of the cluster.

- A division event is triggered by inserting an edge across the centre of the bubble nearest the centre of the cluster, giving two bubbles each with half the area.

In each case we re-equilibrate the structure; T1s occur as part of the disappearance event, but rarely during the equilibration. We record the position of the bubble centres (defined for convenience as the average of the vertex positions) and the bubble pressures before and after the distortion, denoted $x_{i}, y_{i}, p_{i}$ with $i=0,1$.

\section{Results}

\subsection{Bubble displacement}

For free clusters, the displacement vectors of the bubble centres after each event are shown in Fig. 3. As expected, the disappearance event has the largest effect (greater displacements).

Since we apply no constraint during the equilibration to prevent rigid body displacements and rotations, we first calculate the average over all bubble centres of the $x$ and $y$ displacement 


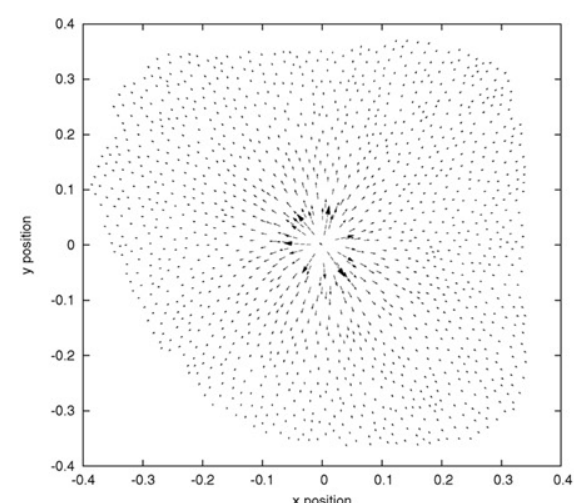

(a)

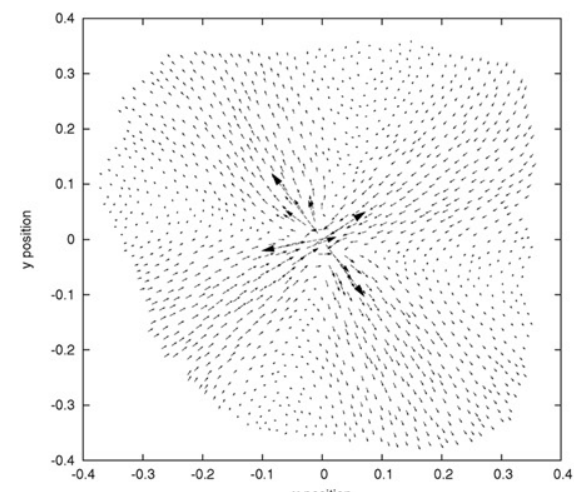

(c)

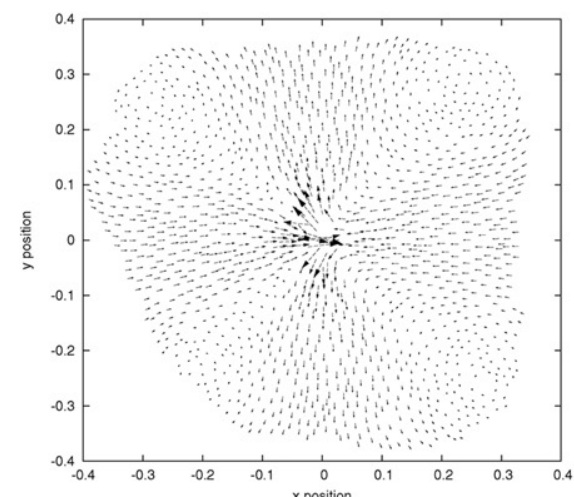

$(\mathrm{e})$

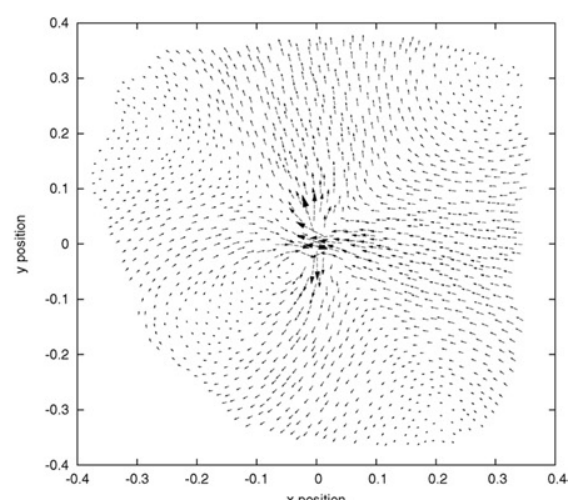

(b)

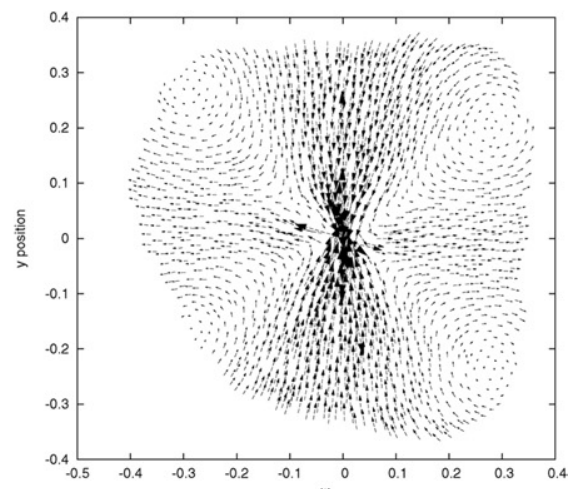

(d)

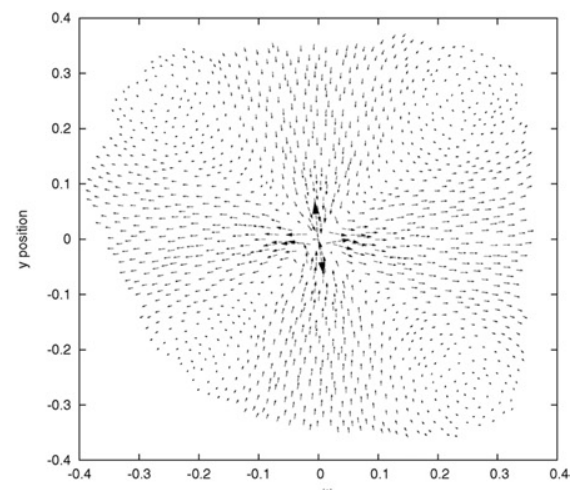

(f)

Fig. 3 The displacement field after (a) 10\% inflation, (b) 20\% edge stretch, (c) T1, (d) disappearance (in which a 6-sided bubble has been removed), (e) coalescence, and (f) division, in a cluster of 1400 bubbles with $\langle A\rangle=3.32 \times 10^{-4}$ and $\operatorname{var}(A) \approx 0.12$. The average displacement and rotation has been subtracted in each case, and the vector length multiplied by a factor of 200 (in a), 100 (in b) or 30 (in c-f).

and the average rotation about the centre of the cluster. These averages are then subtracted from the displacement of each bubble. The results suggest that T1s, disappearance, coalescence, division and edge-stretching events all induce approximately quadrupolar displacement fields, while the inflation event is almost purely radial. Note that the effect of the disappearance event is not isotropic. This is because it triggers several T1s, which break the rotational symmetry. We find no correlation in the magnitude of displacement with the number of sides $n$ of the disappearing bubble.

This information is summarised in Fig. 4, which shows the variation in position of each bubble centre as a function of radial and angular position. The radial displacement
$\Delta r=r_{1}-r_{0}=\sqrt{x_{1}^{2}+y_{1}^{2}}-\sqrt{x_{0}^{2}+y_{0}^{2}}$ decreases with radial distance $r$, and, apart from the inflation event, varies sinusoidally with angle like a quadrupole, $\Delta r \sim \sin \left(2 \theta_{0}\right)$, where $\tan \left(\theta_{0}\right)=y_{0} /$ $x_{0}$ and $\theta_{0}=0$ corresponds to the positive $x$-axis. The angular displacement field $r_{0} \Delta \theta=r_{0}\left(\theta_{1}-\theta_{0}\right)$ is slightly asymmetric but remains approximately quadrupolar.

This quadrupolar angular displacement is not screened and is robust with respect to boundary conditions (Fig. 5). In foams with fixed boundaries, which force the radial displacement to vanish (equivalently, each perturbation interacts with its reflection at boundaries, or virtual image) and with periodic boundary conditions the angular displacement deviates slightly from a sinusoid but is still approximately quadrupolar. 


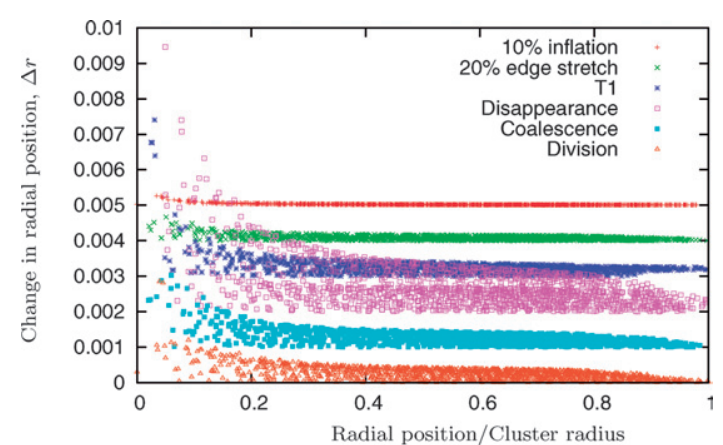

(a)

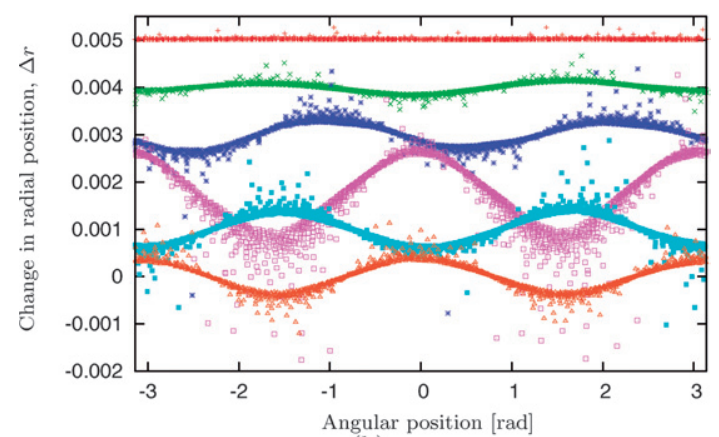

(b)

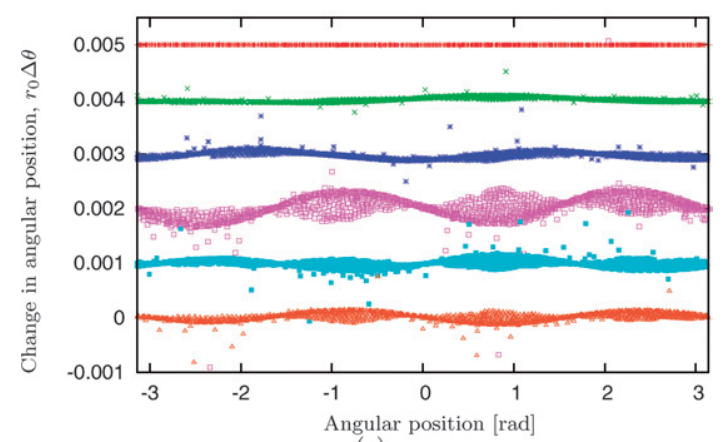

(c)

Fig. 4 Bubble displacement, for the same data shown in Fig. 3, but without multiplicative factors. (a) The magnitude of the radial displacement $|\Delta r|$ as a function of radial position $r_{0}$. (b) The radial displacement $\Delta r$ as a function of angular position $\theta_{0}$. (c) The angular displacement $r_{0} \Delta \theta$ as a function of angular position $\theta_{0}$. Each point represents the movement of a bubble centre. Successive sets of data are shifted vertically a distance 0.001 to distinguish them.

\subsection{Pressure}

The perturbation induces a change in each bubble pressure, $\Delta p=$ $p_{1}-p_{0}$, with respect to the initial foam. We normalise it by $\gamma /$ $\sqrt{ }\langle A\rangle$ to facilitate comparison between different foams. It decreases with radial distance $r_{0}$, as shown in Fig. 6(a).

A small change in the area of a bubble at the centre of the cluster of 150 bubbles, in this case by a factor of $20 \%$, has only a small effect on the pressure differences, which decrease roughly exponentially with radial distance. A similar result was found in a cluster of 1400 bubbles.

All other distortions induce a stronger variation in pressure difference. The largest pressure differences are associated with the disappearance and coalescence events. In the case of a change in edge length, there is little difference between extension and compression; similarly, inflating and shrinking a bubble have the same effect.

The pressure change fluctuates from bubble to bubble, and is either positive or negative. Its standard deviation visibly decreases (Fig. 6(b)). In an ordered cluster, it is possible to define radially concentric shells of bubbles and examine the pressure change in each shell. However, to quantify the pressure differences in disordered foams the shells vary greatly in composition ${ }^{35}$ and it is preferable to bin the data in some manner. We choose a bin width of one typical bubble diameter $D=2 \sqrt{ }(\langle A\rangle / \pi)$, and increase the position of the centre of the bin in intervals of $D / 10$. The average pressure difference $\Delta p$ is calculated in each bin: it is always close to zero beyond the central bubble, indicating that pressure differences are screened effectively. The normalised standard deviation in each bin, $\operatorname{std}(\Delta p)$, directly compares the effect of the different perturbations we apply (Fig. 6(b)).

Remarkably, all perturbations decrease with a similar exponential decay, sometimes over more than two decades. The characteristic length of this decay, which we call the screening length $\ell_{\mathrm{s}}$, is the inverse of the slope:

$$
\frac{1}{\ell_{\mathrm{s}}}=-\frac{\partial \log (\operatorname{std}(\Delta p))}{\partial r}
$$

Quantitative measurements of the screening length are scarce. Szeto et al. $^{13}$ find that correlations in the local foam disorder extend to a distance of three bubble diameters. The available qualitative data ${ }^{6,28,29}$ seem to observe a screening over one or a few bubble diameters. This is what we observe for the different distortions (Fig. 7): despite large changes in several parameters, there is little variation in $\ell_{\mathrm{s}}$, with no clear dependence upon cluster size (data not shown) or disorder. It is in general larger for confined foams and those with periodic boundary conditions.

The choice of $\operatorname{var}(A)$ as the disorder parameter requires some discussion: since the screening is a local effect, we should ideally use a local measure of disorder. It is, however, not clear how to define such a measure.

For all distortions except the bubble inflation (see below), the exponential decrease of $\operatorname{std}(\Delta p)$ crosses over to a plateau near the outer part of the foam. The plateau height is independent of the screening, and instead measures the detection threshold. Thus the cross-over point is almost independent of the screening length. The cross-over length $r_{\mathrm{c}}$ therefore marks the limit where the effect of the perturbation can be detected. We measure it as the intersection of a combined exponential fit to the decrease and a constant value for the plateau (Fig. 6(b)). Its value is very robust (Fig. 8); it is roughly $60 \%$ of the cluster radius independent of perturbation type, boundary conditions and polydispersity. In the present simulations, it corresponds to roughly 5 to 15 bubble diameters. Thus, we could change the volumes of bubbles around the remaining $40 \%$ outer part of the foam without changing the screening length.

The case of inflation in a monodisperse foam is singular (Fig. 9): in contrast to other perturbations, and inflation events in disordered foams, there is no pressure screening. Indeed, the pressure difference increases slightly towards the outside of the cluster. 


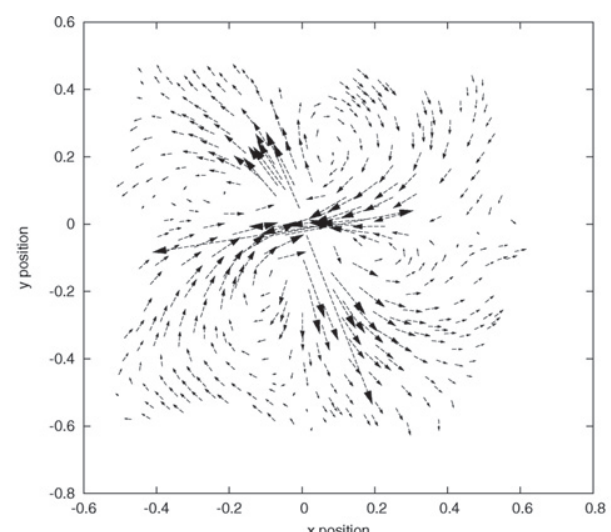

(a)

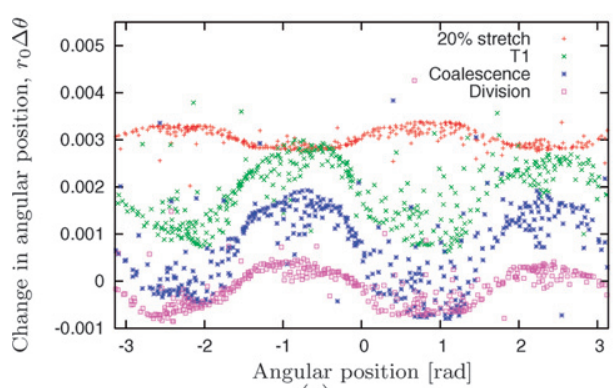

(c)

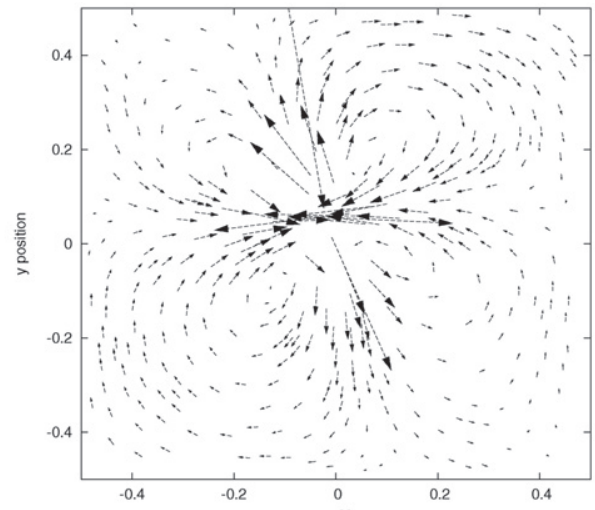

(b)

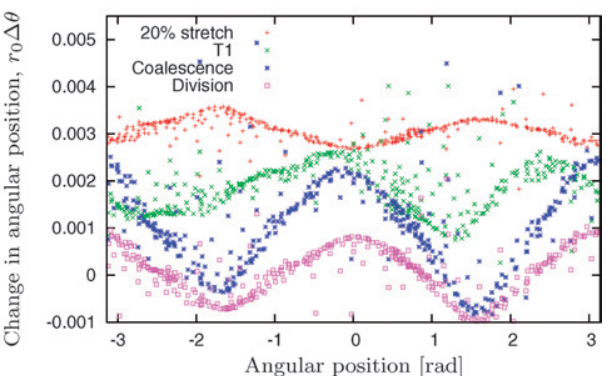

(d)

Fig. 5 Effect of boundary conditions. Bubble displacement field after a T1 in (a) a periodic foam of 400 bubbles and (b) in a confined foam of 383 bubbles. The average displacement and rotation have been subtracted, and the vector length multiplied by a factor of 30 . Angular bubble displacements in the same (c) periodic and (d) confined foams. Successive sets of data are shifted vertically a distance 0.001 to distinguish them.
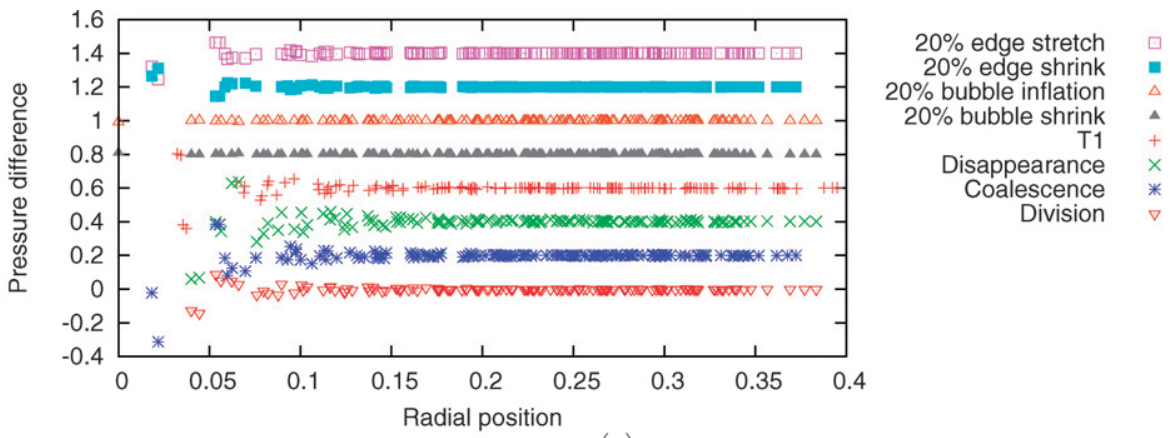

(a)
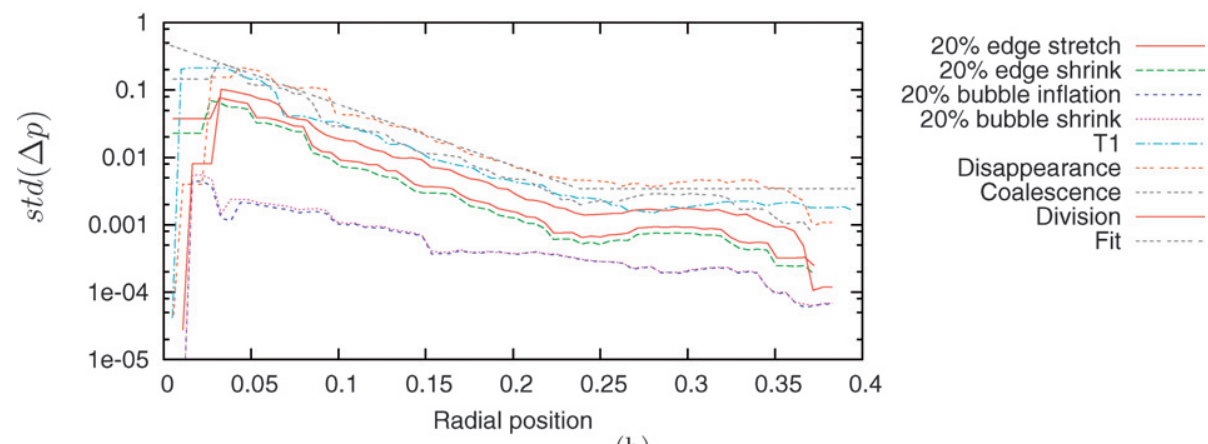

(b)

Fig. 6 (a) The effect on the bubble pressures of each of the distortions in Fig. 2 applied to a disordered monodisperse cluster of 150 bubbles with $A=$ $2.33 \times 10^{-3}$. The effect of an infinitesimal perturbation (top four sets of data) is much less than after one of the discontinuous processes. Each point represents one bubble. Successive sets of data are shifted vertically a distance 0.2 to distinguish them. (b) Semi-log plot of the standard deviation of pressure change (see text) against radial position. The drop-off in the data at large distance is caused by inaccuracy in defining the radius of the periphery of the cluster. The top line, for a disappearance event, is fitted to a piece-wise linear function; the screening length $\ell_{\mathrm{s}}$ is the inverse of the slope of this line in the inner region and the cross-over radius $r_{\mathrm{c}}$ is the radial position at which the plateau is reached. 

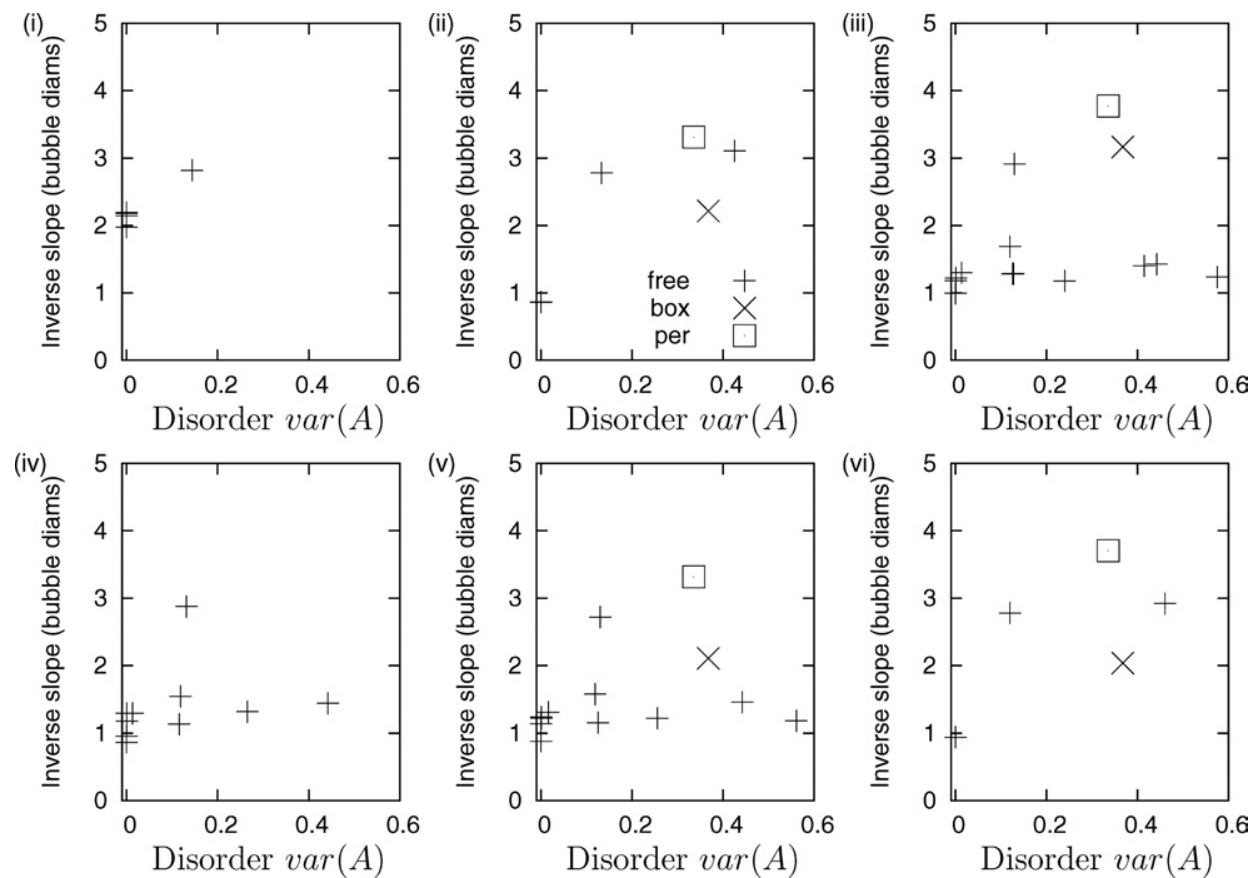

Fig. 7 Screening length $\ell_{\mathrm{s}}$ of $\operatorname{std}(\Delta p)$ (eqn (3)), in units of bubble diameter $D=2 \sqrt{ }(\langle A\rangle / \pi)$, after (i) inflation (ii) an edge stretch, (iii) a T1, (iv) disappearance, (v) coalescence, and (vi) division. Free clusters are denoted with a plus $(+)$, confined foams with a cross $(\times)$ and periodic foams with a box $(\square)$. Error bars are smaller than the point size.

\subsection{Other scalar measures of screening}

The perturbation induces other changes in the structure of the foam, for example each edge may shrink or lengthen to accommodate changes at the centre of the cluster. We justify a posteriori our choice of pressure difference as a measure of screening by comparing it with (i) the change in each bubble's perimeter, $\Delta e$, normalised by the square-root of its area, and (ii) the change in the length of each edge $\Delta \ell,{ }^{6}$ normalised by $\sqrt{ }\langle A\rangle$.

Fig. 10 shows that $\Delta \ell$ decreases quadratically, $\Delta \ell \sim r^{-2}$ over more than a decade in both ordered and disordered foams. $\Delta e$ also decreases quadratically in the disordered cluster, but in the ordered case it shows a more rapid drop, then little change over
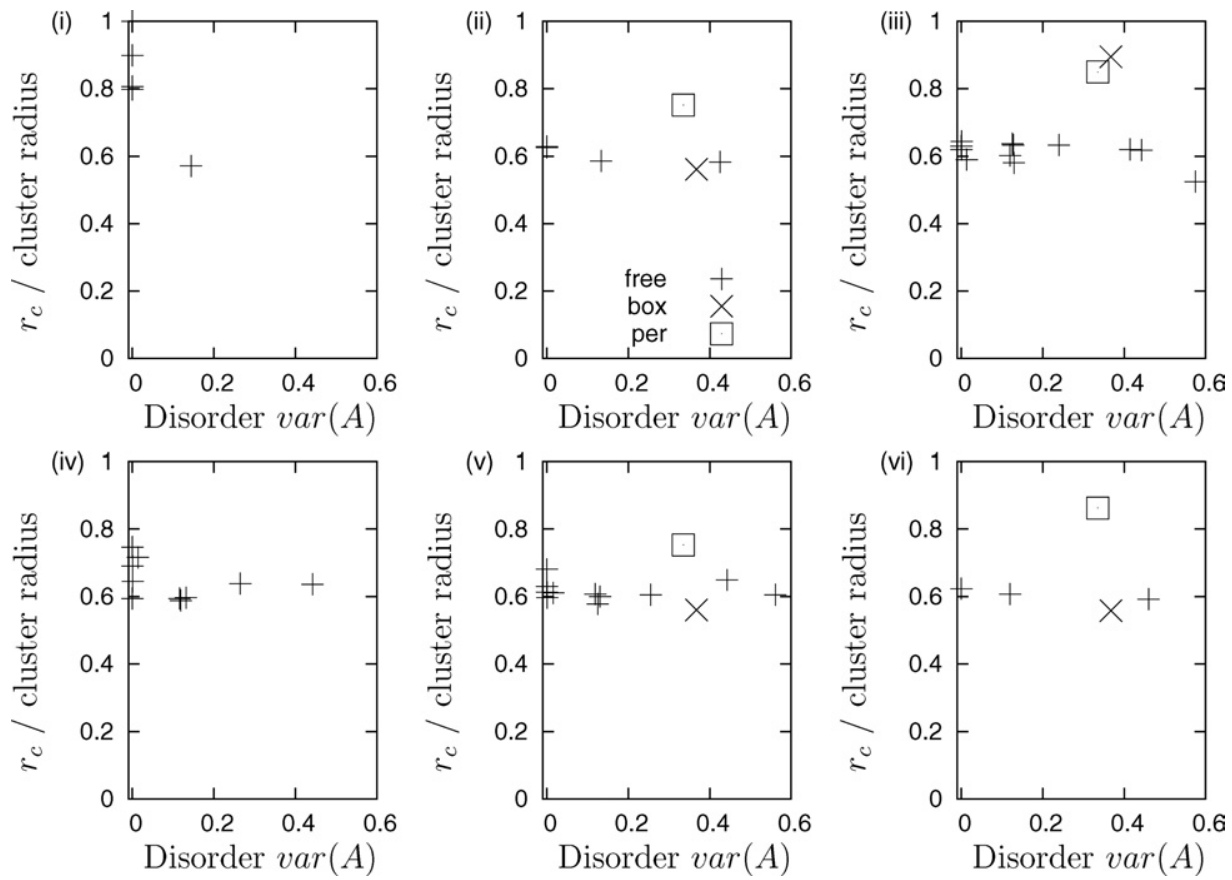

Fig. 8 Cross-over radius $r_{\mathrm{c}}$, in units of foam radius, after (i) inflation, (ii) an edge stretch, (iii) a T1, (iv) disappearance, (v) coalescence, and (vi) division. It is rarely possible to measure it for bubble inflation. Same notation as Fig. 7. Error bars are smaller than the point size. 


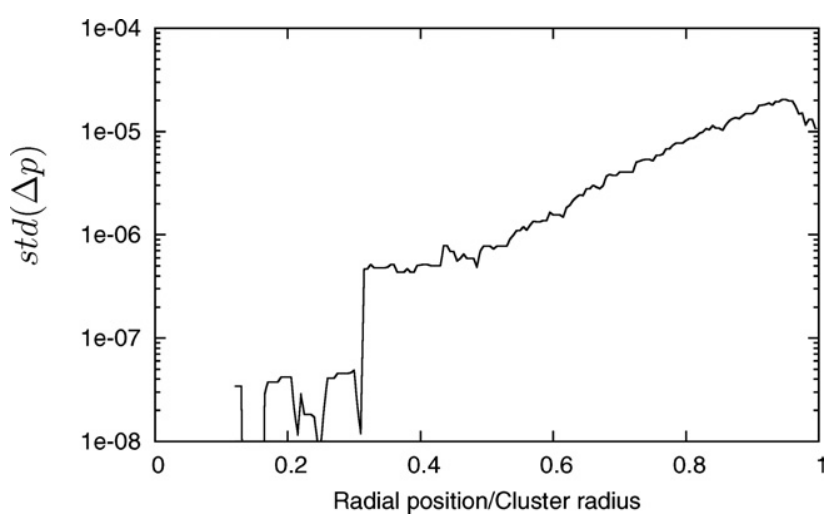

Fig. 9 The effect on the bubble pressures of a $20 \%$ inflation event in a monodisperse ordered cluster of 1261 bubbles. Note the scale, orders of magnitude smaller than Fig. 6(b).

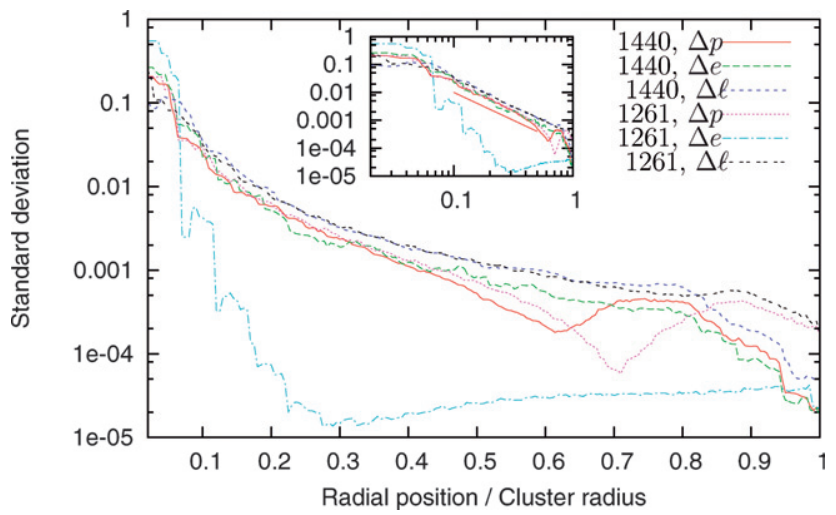

Fig. 10 Semi-log plot of the standard deviation of pressure difference $\Delta p$, perimeter change $\Delta e$ and edge-length difference $\Delta \ell$ against radial position measured in units of cluster radius, comparing data for a $\mathrm{T} 1$ in a disordered cluster of 1400 bubbles with a $\mathrm{T} 1$ in an ordered cluster of 1261 bubbles. The inset shows the same data on $\log -\log$ axes; the solid line has slope -2 .

the outer part of the cluster. Thus neither of these scalar quantities indicates any screening in both ordered and disordered foams, while the pressure change does.

\section{Summary and perspectives}

In simulated dry 2D foams, we have performed continuous and discontinuous perturbations, either isotropic or anisotropic, which conserve or do not conserve the number of bubbles. We have varied polydispersity over a decade and cluster size over almost a decade, with free, periodic or fixed boundary conditions.

The bubble displacements are quadrupolar in all cases except bubble inflation. They extend to the foam boundary and are thus sensitive to boundary conditions.

The pressure change in each bubble fluctuates from one bubble to the next, even for bubbles the same distance from the perturbation. The standard deviation within each of these "shells" decreases exponentially with distance from the perturbation over up to two decades, with a characteristic (screening) length of the order of one average bubble diameter.
At a distance close to $60 \%$ of the foam radius, the standard deviation of pressure difference reaches a plateau. Beyond this distance the perturbation can not be detected.

In wet foams, in which the amount of liquid in the Plateau borders is non-negligible, perturbations such as a T1 are promoted and will probably increase the screening length. ${ }^{36} \mathrm{How}$ the deformation of a bubble affects its neighbours might be crucial in understanding and calculating the shear modulus of a disordered foam ${ }^{37}$ and the role of fluctuations at large scales.

Future work should include the measurement of tensorial quantities such as the deformation; the comparison with displacement and deformation fields predicted by standard elasticity theory ${ }^{26}$ direct comparison with experiments; vectorial anisotropic perturbations, such as can be obtained by moving a point-like defect within the foam (Stokes experiment ${ }^{14}$ ); transition from low disorder to the perfectly ordered case (Fig. 9); and extension to $3 \mathrm{D}$ (where pressure is still a relevant measurement).

\section{Acknowledgements}

We thank S. Courty, E.H.M. Guene, E. Janiaud, J. Käfer, J. Lambert, M. Mancini and other participants in the Grenoble Foam Mechanics Workshop 2008 for stimulation and useful discussions. We thank K. Brakke for his development and maintenance of the Surface Evolver code. SJC thanks the British Council Alliance programme, CNRS and EPSRC (EP/D048397/ 1, EP/D071127/1) for financial support and UJF for hospitality during the period in which this work was conceived.

\section{References}

1 A. M. Kraynik, Annu. Rev. Fluid Mech., 1988, 20, 325.

2 R. Höhler and S. Cohen-Addad, J. Phys.: Condens. Matter, 2005, 17, R1041.

3 P. Marmottant and F. Graner, Eur. Phys. J. E, 2007, 23, 337.

4 J. A. Glazier and D. Weaire, J. Phys.: Condens. Matter, 1867, 4, 1992.

5 D. Weaire and N. Rivier, Contemp. Phys., 1984, 25, 59.

6 F. Elias, C. Flament, J. A. Glazier, F. Graner and Y. Jiang, Philos. Mag. B, 1999, 79, 729 .

7 A. Kabla and G. Debregeas, Phys. Rev. Lett., 2003, 90, 258303.

8 A. Abd el Kader and J. C. Earnshaw, Phys. Rev. E: Stat. Phys., Plasmas, Fluids, Relat. Interdiscip. Top., 1997, 56, 3251.

9 E. M. Guene, J. Lambert et al., in preparation.

10 B. Levitan, Phys. Rev. Lett., 1994, 72, 4057.

$11 \mathrm{M}$. Mancini et al., in preparation.

12 O. Lordereau, PhD thesis, University of Rennes, 2002.

13 K. Szeto, T. Aste and W. Tam, Phys. Rev. E: Stat. Phys., Plasmas, Fluids, Relat. Interdiscip. Top., 1998, 58, 2656.

14 B. Dollet and F. Graner, J. Fluid Mech., 2007, 585, 181.

15 M. E. Rosa and M. A. Fortes, Philos. Mag. A, 1998, 77, 1423.

16 S. Tewari, D. Schiemann, D. Durian, C. M. Knobler, S. A. Langer and A. J. Liu, Phys. Rev. E: Stat. Phys., Plasmas, Fluids, Relat. Interdiscip. Top., 1999, 60, 4385.

17 S. Cohen-Addad and R. Höhler, Phys. Rev. Lett., 2001, 86, 4700.

18 J. Lauridsen, M. Twardos and M. Dennin, Phys. Rev. Lett., 2002, 89, 098303.

19 E. Kolb, C. Goldenberg, S. Inagaki and E. Clement, J. Stat. Mech., 2006, P07017.

20 G. Picard, A. Ajdari, F. Lequeux and L. Bocquet, Eur. Phys. J. E, 2004, 15, 371.

21 Y. Lin, A. Boeker, H. Skaff, D. Cookson, A. D. Dinsmore, T. Emrick and T. P. Russell, Langmuir, 2005, 21, 191.

22 F. Leonforte, A. Tanguy, J. P. Wittmer and J.-L. Barrat, Phys. Rev. B: Condens. Matter, 2004, 70, 014203.

23 A. Tanguy, F. Leonforte and J. L. Barrat, Eur. Phys. J. E, 2006, 20, 355. 
24 C. E. Maloney and A. Lemaître, Phys. Rev. E: Stat. Phys., Plasmas, Fluids, Relat. Interdiscip. Top., 2006, 74, 016118.

25 C. Goldenberg, A. Tangy and J.-L. Barrat, Europhys. Lett., 2007, 80, 16003 .

26 I. Goldhirsch and C. Goldenberg, Euro. Phys. J. E, 2002, 9, 245.

27 C. Goldenberg and I. Goldhirsch, arxiv.org/abs/0711.4243, 2007.

28 R. Farhadifar, J.-C. Röper, B. Aigouy, S. Eaton and F. Jülicher, Curr. Biol., 2095, 17, 2007.

29 S. Courty et al., in preparation.

30 M. A. Fortes, F. Morgan and M. F. Vaz, Philos. Mag. Lett., 2007, 87, 561.
31 K. Brakke. Unpublished, www.susqu.edu/brakke/papers/voronoi. htm, 1986.

32 A. Wyn, I. T. Davies and S. J. Cox, Euro. Phys. J. E, 2008, 28, 81.

33 S. J. Cox and F. Graner, Philos. Mag., 2003, 83, 2573.

34 K. Brakke, Exp. Math., 1992, 1, 141.

35 T. Aste, D. Boosé and N. Rivier, Phys. Rev. E: Stat. Phys., Plasmas, Fluids, Relat. Interdiscip. Top., 1996, 53, 6181.

36 S. J. Cox, M. F. Vaz and D. Weaire, Euro. Phys. J. E, 2003, 11, 29

37 N. P. Kruyt, J. Appl. Mech., 2007, 74, 560. 